\title{
Monte Carlo simulations of the transit dose from amorphous silicon electronic portal images
}

\author{
$\underline{\text { JN. Badel }}{ }^{\mathrm{a}, \mathrm{b} \dagger}$, C. Ginestet ${ }^{\mathrm{a}}$, N. Dufour ${ }^{\mathrm{a}}$ and D. Sarrut ${ }^{\mathrm{a}, \mathrm{b}}$ \\ ${ }^{a}$ Radiotherapy Department, Leon Berard Anticancer Center, Lyon, France \\ ${ }^{b}$ CREATIS (UMR CNRS 5515, Inserm U 630) INSA, Villeurbanne, France
}

\section{Introduction}

Dosimetry with the amorphous silicon (a-Si) electronic portal imaging device is becoming growing popular. The two main applications are the $2 \mathrm{D}$ dose verification, especially for IMRT treatments and the dose received by the patient from the transit portal dose registered during irradiation. In your case, we propose to compare a simulated dose distribution by Monte Carlo (MC) simulation to transit portal dose from the Elekta $i$ ViewGT electronic portal imager. We present here the dosimetric properties of our equipment and the comparisons between computed dose and registered transit dose.

\section{Materials \& Methods}

We investigated the dosimetric properties of $i$ ViewGT mounted on SL-15 accelerator. We computed by MC simulation the transit dose at the level of phosphor screen $\left(\mathrm{Gd}_{2} \mathrm{O}_{2} \mathrm{~S}: \mathrm{Tb}\right)$. We compared pixel values of portal image with transit simulated dose.

Our study was then based on two axes: experimental measures (1) and MC simulations (2).

(1) Three poly-methyl-methacrylate (PMMA) thicknesses: 5, 10 and $20 \mathrm{~cm}$, were placed on the couch of the linear accelerator and positioned such as the half thickness corresponds to the isocentre position. Each thickness has been irradiated by square field sizes of $5 \times 5$, $10 \times 10,15 \times 15,20 \times 20$ and $25 \times 25 \mathrm{~cm}^{2}$ for the nominal energy of $6 \mathrm{MV}$ with a growing monitor unit (from 2 to $250 \mathrm{MU}$ ). The dose rate of the linear accelerator was $200 \mathrm{MU} / \mathrm{min}$. In each acquired portal image we extracted the average pixel value $\bar{P}$ in a region of the interest of $15 \times 15$ pixels (ROI) along the central axis of the beam ${ }^{1}$.

(2) Two Monte Carlo codes were used to compute transit dose ${ }^{2}$ : MCNPX ${ }^{2}$ and $\mathrm{GATE}^{3,4}$ (based and Geant4). The simulated model has been accurately described according to constructor data. The modelled geometry contains a point source which emits photons into a solid angle $\operatorname{OMEGA}(\theta$,$) . The azimuthal angle is sampled uniformly in the range$ from $0^{\circ}$ to $360^{\circ}$. The polar angle $\theta$ is the angle between the incident source particles direction and the central axis of the beam, uniformly sampled between $0^{\circ}$ and $\theta_{\max }$. In this range the photons are uniformly emitted, independently of ${ }_{\theta_{i} i}^{\theta_{\max }}$ and the energy beam photon is sampled according to polyenergetic incident spectra corresponding to $6 \mathrm{MV}$ nominal energy of SL-15.

\section{Results}

Figure 1 shows pixel value-to-MU for studied field sizes and a phantom thickness equals to $5 \mathrm{~cm}$. We obtained a linear relation between pixel value and transit dose with correlation coefficients $\mathrm{R}^{2}>0.99$ per irradiation parameters, which are beam energy, field size and phantom thickness. 
Figure 2 shows values of the computed transit doses by MCNPX $D_{M C N P X}$ and GATE $D_{\text {GATE }}$ normalized by the computed transit dose at $10 \times 10 \mathrm{~cm}^{2}$ and average pixel values ( $\bar{P}$ ) in ROI normalized by the pixel value at $10 \times 10 \mathrm{~cm}^{2}$ in function of field size for a $5 \mathrm{~cm}$ phantom thickness. We observed that the ratios $\frac{\bar{P}}{D_{M C N P X}}$ and $\frac{\bar{P}}{D_{\text {GATE }}}$ were constant in function of the field sizes with a relative standard deviation of $\sim 1.4 \%$ for MCNPX and $\sim 2.6 \%$ for GATE.

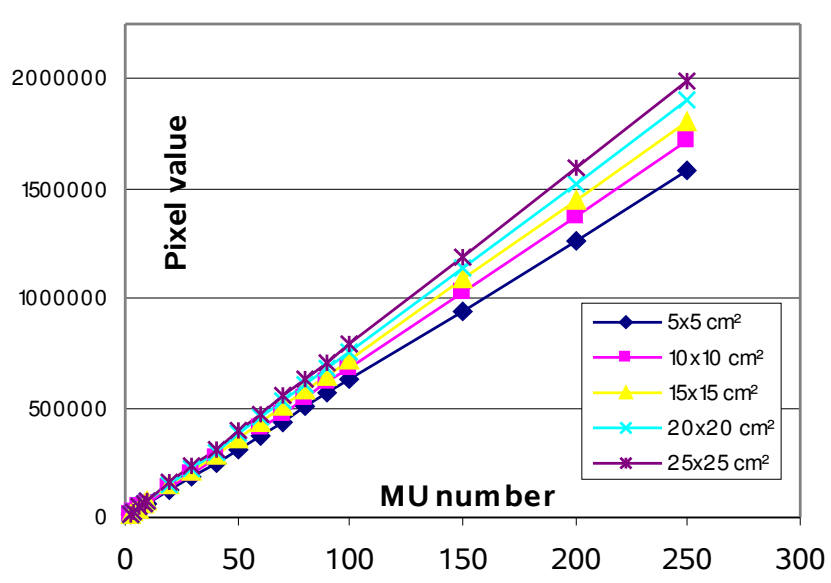

Figure 1: Pixel value for different field sizes in function of the monitor unit (phantom thickness $=65 \mathrm{~cm}$ )
Figure 2: Relative value of simulated transit dose and pixel in function of the field size

\section{Discussion \& Conclusions}

The excellent dosimetric abilities of the $i$ ViewGT have been demonstrated. We have shown that there were as many linear relations as there were irradiation parameters. Moreover, our computed deposition dose by simulation is in good agreement with the experiments. These first results allow to compare the portal transit dose with the simulated transit dose on axis of the beam. Works are ongoing to study a $2 \mathrm{D}$ distribution dose by simulation to compare computed dose with pixel value in a point off-axis.

\section{References}

†Corresponding author: badel@lyon.fnclcc.fr

${ }^{1}$ E.E. Grein et al. "An investigation of a new amorphous silicon electronic portal imaging device for transit dosimetry," Med. Phys. 29, 2262-2268 (2002).

${ }^{2}$ J. V. Siebers, J. O. Lung Ko, and P. J. Keall "Monte Carlo computation of dosimetric amorphous silicon electronic portal images," Med. Phys. 31, 2135-2145 (2004)

${ }^{3}$ L. S. Waters “MCNPX ${ }^{\mathrm{TM}}$ User's Manual, version 2.3.0,” LA-UR-02-2607 Los Alamos, NM: Los Alamos National Laboratory (2002).

${ }^{4}$ S. Jan et al. "GATE: a simulation toolkit for PET and SPECT," Phys. Med. Biol. 49 4543-4561 (2004).

${ }^{5}$ GATE website: http://www-lphe.epfl.ch/GATE/ 